\title{
Luciferase Immunoprecipitation Systems immunoassay is a sensitive, rapid method to detect allergen component-specific IgE
}

Adora A. Lin, MD, PhD ${ }^{\mathrm{a}}$, Natalia S. Perez ${ }^{\mathrm{a}}$, BS, Pamela A. Frischmeyer-Guerrerio ${ }^{\mathrm{b}}, \mathrm{MD}, \mathrm{PhD}$, Thomas B. Nutman, $\mathrm{MD}^{\mathrm{a}}$

${ }^{a}$ Helminth Immunology Section, Laboratory of Parasitic Diseases, and ${ }^{b}$ Food Allergy Research Unit, Laboratory of Allergic Diseases, National Institute of Allergy and Infectious Diseases (NIAID), National Institutes of Health (NIH), Bethesda, MD

Corresponding author/Present address: Adora Lin, MD, PhD, Division of Allergy and Immunology, Children's National Hospital, 111 Michigan Ave NW, Washington, DC 20009.

Telephone: 202-476-3725. Fax: 202-476-6699. Email: aalin@childrensnational.org

\section{Conflicts of interest: None}

Funding source: This study was supported by the Division of Intramural Research, National Institute of Allergy and Infectious Diseases, National Institutes of Health.

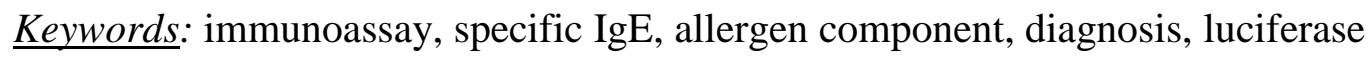
immunoprecipitation systems

Abbreviations/acronyms: LIPS: Luciferase immunoprecipitation systems; Ruc: Renilla luciferase; LU: Light units; ROC: Receiver operating characteristic

Word count: 942

Figures: 1

Tables: None 


\section{Abstract}

Current assays to detect allergen-specific IgE have constraints related to obtaining pure, conformationally active allergen, variability in allergen extracts, sample volume required, and turnaround time. The luciferase immunoprecipitation systems (LIPS) immunoassay is a fast, sensitive assay created using recombinant antigens that requires a low specimen volume. These assays can also be easily modified to detect multiple antigens and antibody isotypes. Here, we demonstrate the use of LIPS assays as an innovative method to quantitatively measure allergen component-specific IgE in small sample volumes. Sera from healthy volunteers, helminthinfected adults, and peanut-allergic children were screened for IgE to cat using ImmunoCAP. These samples were also measured for IgE against Fel d 1 using LIPS. LIPS signal correlated to cat IgE levels with $r_{\mathrm{S}}=0.6204, \mathrm{p}<0.001$. The LIPS signal : noise ratio differed significantly between cat IgE- samples and cat IgE+ samples with values $>0.5 \mathrm{kU} / \mathrm{L}$, with the ability to differentiate cat IgE - individuals from cat IgE+ individuals with 85\% sensitivity and 76\% specificity. Given their rapidity, efficiency, sensitivity, and quantitation over a broad dynamic range, LIPS immunoassays can be a robust and flexible tool with potential uses in allergy research, diagnostics, and treatment. 


\section{Introduction}

Current laboratory methods to determine the presence of circulating allergen-specific IgE involve the use of crude biological extracts or purified recombinant allergens. ${ }^{1}$ Extracts may differ in composition due to difficulties in obtaining a pure, raw material, and/or differences in processing, ${ }^{1,2}$ and recombinant allergens are difficult to obtain in biologically relevant, conformationally active forms. ${ }^{2}$. These limitations with the allergen can lead to variable results in assays. ${ }^{1}$ In addition, challenges arise in patients undergoing evaluation for multiple allergens with restrictions on blood draw volume, such as children. All of these constraints indicate a need for an efficient and sensitive assay using small sample volumes and recombinant proteins. The luciferase immunoprecipitation systems (LIPS) immunoassay has been shown to be an effective method of detection of autoantibodies and antibodies against infectious agents with high sensitivity and specificity. ${ }^{3}$ Advantages of this method over other assays for antibody detection include fast turnaround time, the use of recombinant antigens without elaborate purification schemes, and ease of optimization. ${ }^{3}$ Here, we demonstrate the power of synthetic biology coupled with the LIPS immunoassay as a novel, rapid method to detect allergen-specific IgE in low-volume samples.

\section{Methods}

\section{Clinical samples}

Sera were obtained from healthy volunteers $(n=76)$, helminth-infected adults $(n=26)$, and peanutallergic children $(\mathrm{n}=12)$. All sera were obtained under NIH IRB-approved protocols (99-CC-068, 88-I-83 and15-I-0162), and written informed consent was obtained from all subjects or their parents/legal guardians. Samples were screened for IgE to cat using ImmunoCAP. Cat IgE levels ranged from 0 to $>100 \mathrm{kU} / \mathrm{L}$. 
LIPS assay

LIPS immunoassays were performed as described previously. ${ }^{3}$ Briefly, $5 \mu$ l of patient

44 sera were combined with an optimized amount ( 1 million light units [LU]) of Renilla

45 luciferase-Fel d 1 fusion protein extracts in a 96-well microtiter plate and incubated for 10

46 minutes. Resulting IgE-fusion protein complexes were immunoprecipitated by incubation with

47 anti-IgE beads in a 96-well filter plate. Following several washes to remove unbound fusion

48 proteins, LU were measured on a Berthold LB 960 Centro luminometer (Berthold Technologies,

49 Oak Ridge, TN) using a coelenterazine substrate mixture (Promega, Madison, WI). All samples

50 were run in duplicate.

51

$52 \quad$ Fusion protein constructs

53 Renilla luciferease fusion protein constructs were synthesized for the Fel $\mathrm{d} 1$ component of cat

54 allergen by GenScript Biotech (Piscataway, NJ) using the amino acid sequence previously

55 published ${ }^{10}$. Codons were optimized for expression in mammalian cells. Constructs were

56 transfected into FreeStyle ${ }^{\mathrm{TM}}$ 293-F cells (ThermoFisher, Waltham, MA). Fusion proteins were

57 isolated after 48-72 hours of cell culture by cell lysis in lysis buffer composed of $50 \mathrm{mM}$ Tris,

$58 \mathrm{pH}$ 7.5, $100 \mathrm{mM} \mathrm{NaCl}, 5 \mathrm{mM} \mathrm{MgCl2,} \mathrm{1 \%} \mathrm{Triton} \mathrm{X-100,} \mathrm{50 \%} \mathrm{glycerol} \mathrm{and} \mathrm{protease} \mathrm{inhibitors.}$

59 Anti-IgE beads were created for use in the LIPS assay by coupling goat anti-human IgE

60 (SeraCare KPL, Gaithersburg, MD) to Ultralink beads (ThermoFisher, Waltham, MA).

61

62 Statistical analyses 
63 Figures and statistical analyses, Receiver Operating Characteristic (ROC) curve analysis, and

64 correlations (Spearman’s rank), were done using Prism 6.0 (GraphPad Software, Inc., San Diego,

65 CA). The nonparametric Mann-Whitney test was used to estimate differences in LIPS signal

66 between two groups.

67

\section{Results}

For Fel d 1 LIPS, there was no significant difference between the signal: noise ratio for cat IgE - samples and cat IgE+ samples with values of $0.36-0.49 \mathrm{kU} / \mathrm{mL}$. However, the signal :

71 noise ratio differed significantly between cat IgE- samples and cat IgE+ samples with values >

$720.5 \mathrm{kU} / \mathrm{mL}$ and encompassed a broad dynamic range (Figure 1A, $p<0.02$ ). LIPS signal

73 correlated to cat IgE levels with $r_{\mathrm{S}}=0.6204, p<0.001$ (Figure 1B). ROC analysis gave an area

74 under the curve of $0.7929(p<0.001)$, which increased to $0.8455(p<0.001)$ if cat IgE+ samples

75 were defined as being $\geq 0.5 \mathrm{kU} / \mathrm{mL}$. A threshold of $1647 \mathrm{LU} / \mu \mathrm{l}$ distinguished cat IgE -

76 individuals from cat IgE+ individuals with 85\% sensitivity and 76\% specificity. Assays were

77 completed in less than one hour.

\section{Discussion}

These results demonstrate that LIPS immunoassays can quantify component-specific IgE

81 in small volumes of human serum. Although our sample cohort was relatively small and

82 heterogeneous, the sensitivity and specificity of the LIPS assay for identifying cat IgE was

83 similar to that reported for aeroallergen skin prick testing. ${ }^{4}$ We did not have information

84 regarding skin prick sensitivity to cat or clinical symptoms following cat exposure for our cohort; 
85 it is possible that the efficacy of LIPS would be increased if our analysis had been able to include

86

87

88

89

90

91

92

93

94

95

96

97 these parameters.

For Fel d 1, the LU correlated with cat IgE levels by ImmunoCAP. This is consistent with studies showing that Fel $\mathrm{d} 1$ is a reliable marker for cat sensitization and has high sensitivity and specificity for clinical reactivity to cat. ${ }^{5}$ For patients with positive cat IgE but low LIPS signal, it is either because their IgE specific for Fel d 1 is below the limits of detection of the assay (possible, but unlikely), or rather their IgE response is specific for a different Fel d component. Most other aeroallergens do not have a component with the same dominance over others that Fel $\mathrm{d} 1$ has in cat; ${ }^{2}$ thus, using a single component to determine sensitivity may not be clinically useful. However, LIPS is a high throughput system where responses to multiple target antigens can be performed, ${ }^{6}$ and components could be combined in a single assay to more accurately determine allergen sensitivity. Indeed, we have piloted detection of IgE specific for 5 peanut components (data not shown).

The LIPS immunoassay offers a sensitive and efficient method to both qualitatively and quantitatively determine an individual's sensitization patterns to major components of allergens, using substantially less serum than is typically sent for a single allergen-specific IgE. This is of importance in vulnerable populations, such as children or individuals with anemia or reduced cardiac output, who require evaluation for multiple allergens and allergen components and have limitations on blood collection volumes. LIPS could have major utility in food allergy, where component-resolved diagnostics are clinically available and are applied in determining likelihood of clinical reactivity. ${ }^{7}$ With increasing interest in the development of component-resolved immunotherapy, one can also envision LIPS as part of the process in creating customizable immunotherapy based on an individual's sensitization profile. 
109 other antibody isotypes, such as IgG4 or IgA, specific to a particular allergen or allergen

110 component, simply by changing the isotype specificity of the antibody used to precipitate

111 antibody-protein complexes. ${ }^{8}$ The LIPS assay may also be modified to evaluate for

112 quantification of components themselves, ${ }^{9}$ which would have utility in determining component

113 levels in foods, environmental samples, and allergen extracts.

114 In summary, due to their sensitivity, use of small amounts of sample, and rapidity, LIPS

115 immunoassays can be a powerful and malleable tool with numerous uses in allergy research, 116 diagnostics, and treatment. 


\section{REFERENCES}

1. Hamilton RG. Clinical laboratory assessment of immediate-type hypersensitivity. J Allergy Clin Immunol. 2010;125(2 Suppl 2):S284-96.

2. Cromwell O, Hafner D, Nandy A. Recombinant allergens for specific immunotherapy. J Allergy Clin Immunol. 2011;127(4):865-72.

3. Burbelo PD, Ching KH, Klimavicz CM, Iadarola MJ. Antibody profiling by Luciferase Immunoprecipitation Systems (LIPS). J Vis Exp. 2009(32).

4. Nevis IF, Binkley K, Kabali C. Diagnostic accuracy of skin-prick testing for allergic rhinitis: a systematic review and meta-analysis. Allergy Asthma Clin Immunol. 2016;12:20. 5. Gronlund H, Saarne T, Gafvelin G, van Hage M. The major cat allergen, Fel d 1, in diagnosis and therapy. Int Arch Allergy Immunol. 2010;151(4):265-74.

6. Burbelo PD, Leahy HP, Iadarola MJ, Nutman TB. A four-antigen mixture for rapid assessment of Onchocerca volvulus infection. PLoS Negl Trop Dis. 2009;3(5):e438.

7. Santos AF, Brough HA. Making the Most of In Vitro Tests to Diagnose Food Allergy. J Allergy Clin Immunol Pract. 2017;5(2):237-48.

8. Burbelo PD, Ramanathan R, Klion AD, Iadarola MJ, Nutman TB. Rapid, novel, specific, high-throughput assay for diagnosis of Loa loa infection. J Clin Microbiol. 2008;46(7):2298-304. 9. Drame PM, Meng Z, Bennuru S, Herrick JA, Veenstra TD, Nutman TB. Identification and Validation of Loa loa Microfilaria-Specific Biomarkers: a Rational Design Approach Using Proteomics and Novel Immunoassays. MBio. 2016;7(1):e02132-15.

10. Kaiser L, Velickovic TC, Badia-Martinez D, Adedoyin J, Thunberg S, Hallen D, et al. Structural characterization of the tetrameric form of the major cat allergen Fel d 1. J Mol Biol. 2007;370(4):714-27. 


\section{FIGURE LEGEND}

2 FIGURE 1. LIPS assay detects Fel d 1-specific IgE. Top panel: Fel d 1 LIPS signal: noise ratio

3 in cat $\operatorname{IgE}-(n=74)$ and cat $\operatorname{IgE}+$ individuals, subdivided into cat $\operatorname{IgE}<0.5 \mathrm{kU} / \mathrm{mL}(n=7), 0.5$ -

$410 \mathrm{kU} / \mathrm{mL}(n=24)$, and $>10 \mathrm{kU} / \mathrm{mL}(n=9)$. Significant differences were found amongst all

5 groups (Kruskal-Wallis test) and between all pairs except for cat IgE- and cat IgE+ $<0.5$ (Mann-

6 Whitney test). Bottom panel: Light units per $\mu$ l of serum vs. cat $\operatorname{IgE}+1$ (to accommodate $\log$

$7 \quad$ scale). $n=91$. 
Figure 1
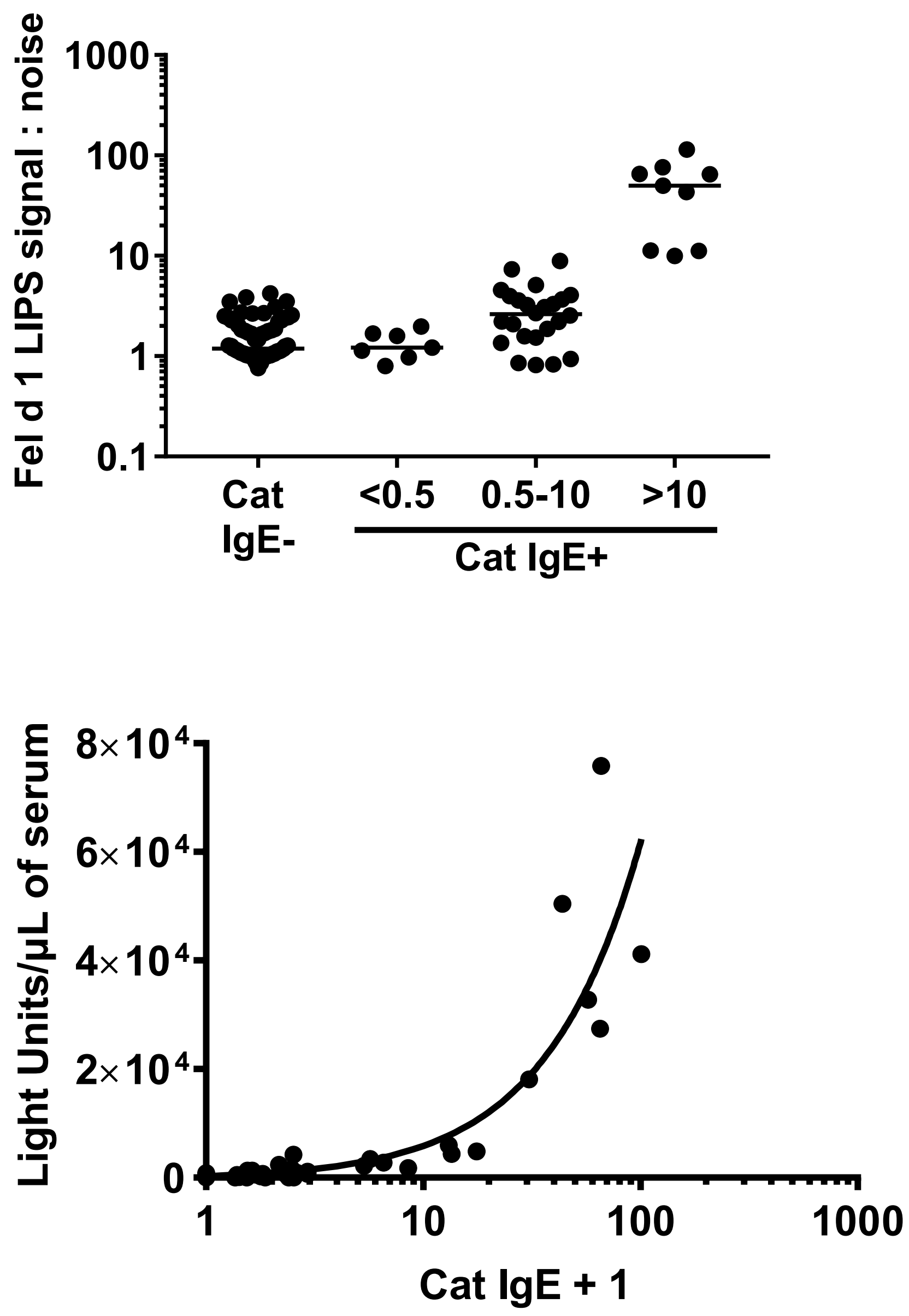